\title{
Study on the Early Warning of Three-phase Unbalance Online Monitoring Based on Data Mining
}

\author{
Chen Gao ${ }^{a}$, Zhong'an Ding ${ }^{\mathrm{b}}$ and Wuxiao Chen ${ }^{\mathrm{c}}$ \\ Electric Power Research Institute of State Grid Fujian Electric Power Company Limited, Fuzhou \\ 350000, China.

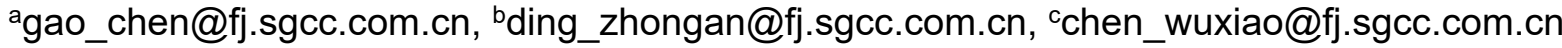

Keywords: data mining, three-phase unbalance, urgent index.

\begin{abstract}
In the process of power system operation, three-phase unbalance has become normal. Based on the limitation of the filter rules on the three-phase unbalance, this article builds the discriminate model of it, firstly, eliminate abnormal data and restore the missing data through analyzing the real data distribution of three-phase unbalance ; then utilize the difference between the maximum phase current and the minimum phase current and study out a evaluation function on the urgent degree of three-phase unbalance, then qualitatively evaluate the urgent degree of reforming three-phase unbalance of different distribution transformer, and calculate its urgent index number. Based on these analyses, we can provide a more scientific and effective way of judging three-phase to guide the reform of unbalanced three-phase distribution transformer area for the workers in the power system.
\end{abstract}

\section{Introduction}

The transformer which charged with an important task of transforming and distributing electric energy is the hub of the distribution transformer area and circuit provides the channel for power transmission. A scientific and reasonable circuit structure guarantees power to transmit in a safe, high-quality and low-loss way. Three-phase unbalance is not only the basis to evaluate the reasonability about circuit structure of distribution transformer area, but also the basic requirement to ensure a transformer running normally. Whether three-phase is balanced or not relates to the reliability and stability as well as loss rate of the power supply.

Currently, three-phase unbalance has become normalcy in the process of running the grid. It would lead transformer lose more, zero-sequence current heavier and local temperature of mental too high. Furthermore, circuit as well as transformer could be burned out. Jianghao and Wangmiao (2008) gave the viewpoint that three-phase unbalance would increase energy loss of power grid in accordance with the analysis between three-phase load unbalance and low voltage grid loss. Guofeng and his cooperators (2007) came up with a root-mean-square current method for computing the theoretical value of low voltage grid loss. They analyzed circuit loss induced by three-phase unbalance, then put forward a formula to explain what existed between the loss and the unbalance. Additionally, Cui Jiangming (1999), Bihua (2010) and their partners elaborated the hazard that three-phase unbalance would bring to a transformer. What's more, three-phase unbalance is obviously relevant to some index outliers such as low voltage and low power. Therefore, a well-targeted reform is quite significant to three-phase unbalance.

In terms of three-phase unbalance discrimination, $\mathrm{Wu}$ Wenchuan and Zhang Boming (2002) proposed a unified analysis method by using a multi-port mixed phase compensation technique to discriminate three-phase unbalance power flow and fault calculation. Wang Xiting and Huang lanfeng (2007) analyzed transformer direct-current resistance caused by three-phase unbalance to find out the reason of the failure and then gave the common fault diagnosis method. T H, Chen M S, Lee W J, et al (1992) adopted nodal admittance matrix and iterative compensation act to test malfunction. The popular filtering rules of three-phase unbalance are based on some experience and practical foundation, but there exist limitations as well. For instance, some severe cases have not been 
incorporated into the category, and systematic discriminant model has not been established so that we are unable to make the evaluation about three-phase unbalance severity. These methods are more complicated to calculate and less efficient to operate.

In view of current filtering rules of three-phase unbalance are not sound, this article treats the discriminant model establishment and the severity test as the core. In the first place, it removes abnormal values and restores missing values based on analyzing the actual data distribution of three-phase unbalance. Then this article studies out an evaluation function which can reflect the urgent level of three-phase unbalance and give star-rated evaluation by using the difference between maximum phase current and minimum phase current so that grid workers can be provided a more scientific as well as efficient method to discriminate three-phase unbalance and direct relevant reform to distribution area. Empirical findings indicate that this method has not only a higher precision but also a better guidance and can be widely used.

\section{Modeling}

\subsection{Evaluation of the Maximum Phase Current}

The severity of three phase unbalance and the reformed urgent index will increase along with an added maximum phase current since an approximately positive correlation between current and grid load. In order to ensure certain electrical load, each point of maximum phase current must be bigger than $1.5 \mathrm{~A}$ based on experience, otherwise the severity of three phase unbalance would decrease in the condition of unobvious electrical load. So maximum phase current as one of explanatory variables should be incorporated into the evaluation function of three-phase unbalance urgent index. Define:

$$
\begin{aligned}
& I_{i}^{\max }=\max \left(I_{A i}, I_{B i}, I_{C i}\right) \\
& I_{i}^{\min }=\min \left(I_{A i}, I_{B i}, I_{C i}\right)
\end{aligned}
$$

Where, $i=1,2, \cdots, n$. At the same unbalanced level, the bigger $I_{i}^{\max }$ is, the more harmful power distribution would get. As a result, the reformed urgent index will also be magnified. The urgent index factor of maximum phase current is expressed by the following equation:

$$
\kappa_{1}\left(I_{i}^{\max }\right)=\frac{1}{1+e^{\alpha_{1} I_{i}^{\max }+\alpha_{2}}}
$$

According to experience, we assume that: $I_{i}^{\max }=1, \kappa_{1}\left(I_{i}^{\max }\right)=0.2$ and $I_{i}^{\max }=2, \kappa_{1}\left(I_{i}^{\max }\right)=0.8$; The equation can be determined by the given values:

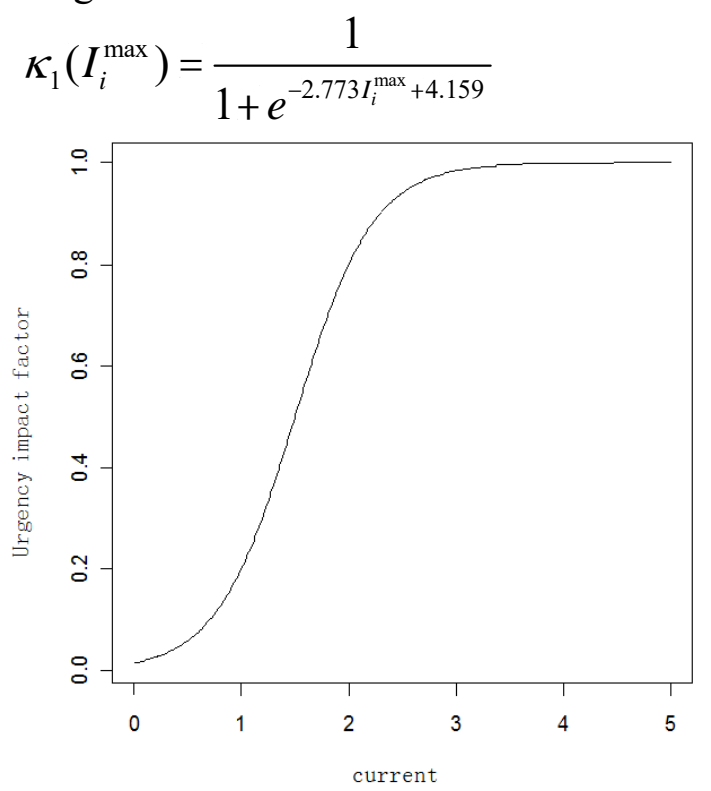

Fig 1. The impact factor curve graph of maximum phase current 


\subsection{Maximum Minimum Principle}

As the most important impact factor, the difference between maximum phase current and minimum phase current, of course, should be subordinated to urgent index evaluation function.

According to the definition, three-phase current unbalance value $=$ (maximum phase current-minimum phase current)/ maximum phase current*100\%, that is:

$$
\eta_{i}=\frac{\left|I_{i}^{\max }-I_{i}^{\min }\right|}{I_{i}^{\max }}
$$

In the $\mathrm{n}$ points in one day, theoretically, the more unbalanced points have, the more urgent distribution transformer should maintain. However, they are not simple linear relationship. This article describes their relation by using logistic regression model and define urgent impact factor concretely as follows:

$$
\kappa_{2}(j)=\frac{1}{1+e^{\beta_{1} j+\beta_{2}}}
$$

Where, $j$ is the number of element in assembly $w, w \subseteq[1, n]$ is the subscript assembly that three-phase current unbalance belonging to $\left[\eta_{a}, \eta_{b}\right]$ correspond to during acquisition time.(For example: if $\eta_{i} \in\left[\eta_{a}, \eta_{b}\right], i \in w, \eta_{a}=60 \%, \eta_{b}=90 \%$ ).Based on historical data , when $j_{1}=12, k_{2}(j)=0.2$; when $j=24, k_{2}(j)=0.7$.So the second contribution function can be expressed by the following formula:

$$
\kappa_{2}(j)=\frac{1}{1+e^{-0.223 j+4.067}}
$$

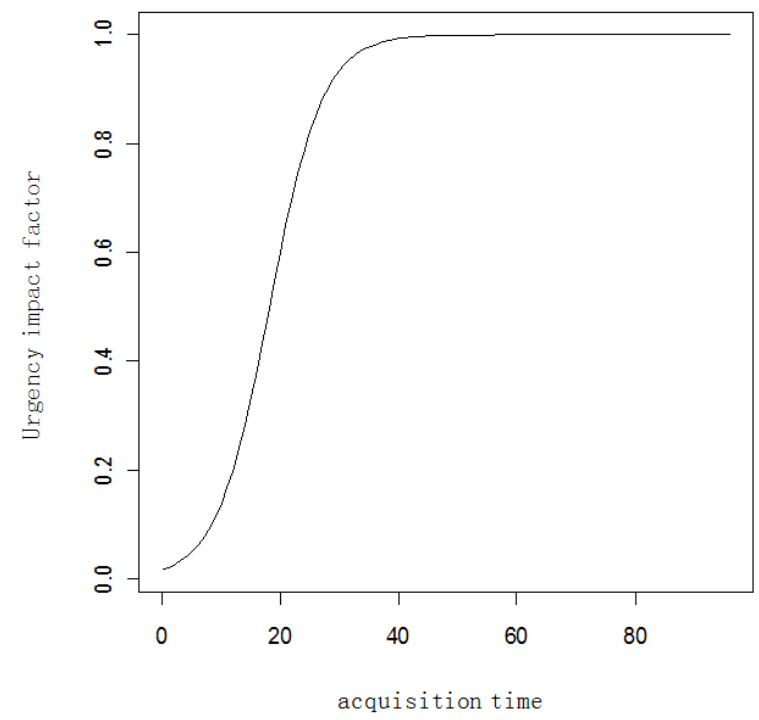

\subsection{Urgent Index}

Fig 2. Urgency impact factor curve graph during acquisition time

According to above analysis, the urgent index of three-phase unbalance is defined as follows:

$$
\zeta=\kappa_{2}(j) H_{k<20}\left[\frac{2}{5 j} \sum_{i \in \omega} \kappa_{1}\left(I_{i}^{\max }\right)\left(\frac{\eta_{i}}{\eta_{b}-\eta_{a}}-\frac{\eta_{a}}{\eta_{b}-\eta_{a}}\right)+\frac{3}{5}\right]+H_{k \geq 20}
$$

Where: $H_{x}$ is indicative function; $k$ is the number of acquisition time that three-phase current unbalance is bigger than $\eta_{b} ; \zeta \in[0,1]$ is the urgent level of distribution transformer reform. When $\zeta=1$, this reform is most urgent and the transformer need to be maintained as soon as possible.

The equation can compute daily three-phase unbalance urgent index of distribution transformer. Define: when $\zeta<0.3$, the three-phase can be considered balance. If there exist ten or more days during one month, the three-phase can be considered unbalance. Assume that $p$ days urgent index is: $\zeta_{1}, \zeta_{2}, \cdots \zeta_{p}$ in one month, the comprehensive urgent index is:

$$
\bar{\zeta}=I_{P \geq 10} \frac{1}{p} \sum_{i=1}^{p} \zeta_{i}
$$




\section{Empirical Analysis}

This article randomly selects 10 distribution areas in certain domestic province to analyze their three-phase unbalance. Making use of traditional algorithm given by the province and new algorithm given by this article to discriminate respectively, the result is expressed under the following table:

Table 1. Analysis table of urgent index

\begin{tabular}{|c|c|c|c|}
\hline $\begin{array}{l}\text { distribution } \\
\text { area number }\end{array}$ & $\begin{array}{l}\text { urgent } \\
\text { index }\end{array}$ & $\begin{array}{l}\text { traditional algorithm to } \\
\text { discriminate }\end{array}$ & new algorithm to discriminate \\
\hline 1 & 0.6719 & normal & abnormal and need reformed \\
\hline 2 & 1.0000 & abnormal & $\begin{array}{l}\text { abnormal and maybe one-phase } \\
\text { disconnection }\end{array}$ \\
\hline 3 & 0.8587 & abnormal & abnormal and need reformed urgently \\
\hline 4 & 1.0000 & abnormal & $\begin{array}{l}\text { abnormal and maybe one-phase } \\
\text { disconnection }\end{array}$ \\
\hline 5 & 0.7575 & normal & abnormal and need reformed \\
\hline 6 & 0.7561 & abnormal & abnormal and need reformed \\
\hline 7 & 0.7559 & abnormal & abnormal and need reformed \\
\hline 8 & 0.7559 & normal & abnormal and need reformed \\
\hline 9 & 0.7554 & abnormal & abnormal and need reformed \\
\hline 10 & 0.7438 & abnormal & abnormal and need reformed \\
\hline
\end{tabular}

As presented in the above table, traditional method can only discriminate whether three-phase unbalance exists in distribution transformer but is not able to discriminate the severity. Based on relevant analysis, this article, however, explicitly raises urgent index to elaborate its severity. Furthermore, some abnormal distribution areas would not have passed the test according to traditional algorithm, but the algorithm given by this article can find out these areas exactly, which will improve the efficiency of grid.

\section{Conclusion}

This article analyzes the data feature of three-phase unbalance and establishes the discriminant model to test three-phase unbalance. In addition, it introduces the urgent index to intuitively explain the severity of three-phase unbalance. Empirical findings indicate that this method not only has a better effect but also a higher precision and can be widely used.

\section{References}

[1]. Chen T H, Chen M S, Lee W J, et al. Distribution System Short Circuit Analysis-A Rigid Approach[C]. IEEE Trans on Power Systems. Vol.7(1992) No.1, p. 444-450.

[2]. Bi Hua. The harmfulness of distribution transformer with unbalanced three-phase load and relevant design. [J]. High-Technology \& Industrialization. (2010) No.1, p.82-84.

[3]. Cui Jianmin, Wu Xinhua, Guo Yalin. By an accident to know the harmfulness of distribution transformer with unbalanced three-phase load[J]. Shanxi Coal. Vol.19 (1999) No.1, p.26-27.

[4]. Guo Feng, Yao Lina, Liu Heng, Wu Shanshan. Theoretical line loss calculation with three-phase unbalance degree for low-voltage distribution network[J]. Electric Power Automation Equipment. Vol.27 (2007) No.11, p.51-54.

[5]. Jiang Hao, Wang Miao. Analysis on the Influence of Unbalanced Three-phase Loads on LV Line Loss [J]. Power Supply. Vol.25 (2008) No.3, p.68-70.

[6]. Wang Xiting, Huang Fenglan. The Reason and Fault Diagnosis of Transformer Derect Current Resistance [J]. Scientific and Technological Innovation in Heilongjiang. (2007) No.23, p.5-6. 
[7]. Wu Wenchuan, Zhang Boming. A Uniform Approach for Power Flow Analysis and Fault Analysis of Asymmetrical Three-phase Distribution System[J]. Electric Power System Automation. Vol.26 (2002) No.20, p.50-54. 